\title{
Calculating the Euler characteristic of the moduli space of curves
}

\author{
Bodo Lass \\ Univ Lyon, CNRS, Université Claude Bernard Lyon 1, \\ UMR 5208, Institut Camille Jordan, \\ F-69622 Villeurbanne, France \\ lass@math.univ-lyon1.fr
}

\begin{abstract}
The orbifold Euler characteristic of the moduli space $\mathcal{M}_{g ; 1}$ of genus $g$ smoth curves with one marked point $(g \geq 1)$ was calculated by Harer and Zagier: $\chi\left(\mathcal{M}_{g ; 1}\right)=\zeta(1-2 g)=$ $-B_{2 g} /(2 g)$, where $\zeta$ is the Riemann zeta function and $B_{2 g}$ is the $(2 g)$ th Bernoulli number. We want to make their proof shorter and more combinatorial.
\end{abstract}

\section{Introduction}

Harer and Zagier [15] have shown that calculating the orbifold Euler characteristic of the moduli space $\mathcal{M}_{g ; 1}$ of genus $g$ smoth curves with one marked point is a purely combinatorial problem:

Let us consider a (convex regular) $(2 m)$-gon $(m \geq 1)$, orient each of its $2 m$ edges counterclockwise and label them from 1 to $2 m$ following the orientation. There are

$$
(2 m-1) ! !=(2 m-1) \cdot(2 m-3) \cdots 5 \cdot 3 \cdot 1=\frac{(2 m) !}{2^{m} m !}
$$

perfect matchings on a set of $2 m$ elements providing $(2 m-1) ! !$ possible gluings in pairs of the oriented sides of our $(2 m)$-gon, such that two oppositely oriented arcs become an unoriented edge. Of course the gluing of two oppositely oriented arcs implies the identification of their endpoints reducing the number of vertices from $2 m$ to some integer $v$. The embedded graph (or Riemann surface) obtained in this way has one face (the $2 m$-gon), $m$ edges, $v$ vertices and therefore the Euler characteristic $g$ determined by

$$
v-m+1=2-2 g \quad \Leftrightarrow \quad v+2 g=m+1 .
$$

Of course,

$$
g \geq 0 \Leftrightarrow v \leq m+1 \text { and } v \geq 1 \Leftrightarrow 2 g \leq m .
$$

We suppose that among our $(2 m-1)$ !! possible identifications $\varepsilon_{g}(m)$ give and embedded graph of genus $g$, whereas $\lambda_{g}(m)$ of those embedded graphs of genus $g$ do not contain any vertex of degree 1 or 2 . If every vertex degree has to be at least 3 , our identity $3 v+6 g=3 m+3$ implies the additional inequality

$$
3 v \leq 2 m \Leftrightarrow m \leq 6 g-3 .
$$

Harer and Zagier [15] have shown that the orbifold Euler characteristic of the moduli space $\mathcal{M}_{g ; 1}$ of genus $g$ smoth curves with one marked point $(g \geq 1)$ is equal to

$$
\chi\left(\mathcal{M}_{g ; 1}\right)=\sum_{m=2 g}^{6 g-3}(-1)^{m-1} \frac{\lambda_{g}(m)}{2 m} .
$$


This theorem can be proved using the isomorphism, as real orbifolds, of the decorated moduli space $\mathcal{M}_{g ; 1} \times \mathbb{R}_{+}$and the combinatorial moduli space $\mathcal{M}_{g, 1}^{\text {comb }}$ whose $m$-dimensional part consists of the embedded graphs counted by $\lambda_{g}(m)$ (in order to calculate the orbifold Euler characteristic we have to divide by $2 m$, the number of automorphisms of our $(2 m)$-gons, and to multiply by $(-1)^{\text {codim }}=(-1)^{(6 g-3)-m}=(-1)^{m-1}$ ). Indeed, if we fix a positive real number (which will be the perimeter of our $(2 m)$-gon) for our marked point, then there is a unique quadratic differential (Strebel differential), and we can look at its critical graph. It is embedded, its edges have fixed lengths, and its vertices are the zeroes of the differential, where a zero of order $i$ gives a vertex of degree $i+2 \geq 3$ (see $[20,22,36]$ for more details). In particular, the real dimension of $\mathcal{M}_{g, 1}^{\text {comb }}$ and $\mathcal{M}_{g ; 1} \times \mathbb{R}_{+}$is $6 g-3$ giving a complex dimension of $3 g-2$ for $\mathcal{M}_{g ; 1}$. If $g=1$, the identity (1.5) implies

$$
\chi\left(\mathcal{M}_{1 ; 1}\right)=-\frac{\lambda_{1}(2)}{4}+\frac{\lambda_{1}(3)}{6}=-\frac{1}{4}+\frac{1}{6}=-\frac{1}{12},
$$

because for a 4-gon and a 6-gon, it is necessary to glue each side with its opposite one in order to avoid the creation of vertices of degree 1 or 2 , and in this case we always get $g=1$. For $g \geq 2$, however, calculating $\lambda_{g}(m)$ becomes more complicated, a problem which Harer and Zagier [15] solved in three steps: First, they proved recurrence relations allowing to calculate the numbers $\lambda_{g}(m)$ with the help of the numbers $\varepsilon_{g}(m)$ (not involving restrictions on vertex degrees). Then they established the following beautiful formula.

Theorem 1. (Harer-Zagier formula) For fixed $m \in \mathbb{N}^{*}$ and for every $N \in \mathbb{N}^{*}$ we have

$$
\sum_{g=0}^{\lfloor m / 2\rfloor} \varepsilon_{g}(m) \cdot N^{m+1-2 g}=\sum_{n=1}^{N}\left(\begin{array}{c}
N \\
n
\end{array}\right) \cdot(2 m-1) ! ! \cdot 2^{n-1} \cdot\left(\begin{array}{c}
m \\
n-1
\end{array}\right) .
$$

And finally, they put everything together to obtain their main result.

Theorem 2. The orbifold Euler characteristic of the moduli space $\mathcal{M}_{g ; 1}$ of genus $g$ smoth curves with 1 marked point $(g \geq 1)$ equals

$$
\chi\left(\mathcal{M}_{g ; 1}\right)=\zeta(1-2 g)=-\frac{B_{2 g}}{2 g},
$$

where $\zeta$ is the Riemann zeta function and $B_{2 g}$ is the $(2 g)$ th Bernoulli number, i.e. the coefficient of $z^{2 g}$ in the expansion

$$
\begin{gathered}
\quad \frac{z}{e^{z}-1}=1-\frac{1}{2} z+B_{2} \frac{z^{2}}{2 !}+B_{4} \frac{z^{4}}{4 !}+B_{6} \frac{z^{6}}{6 !}+B_{8} \frac{z^{8}}{8 !}+B_{10} \frac{z^{10}}{10 !}+B_{12} \frac{z^{12}}{12 !}+B_{14} \frac{z^{14}}{14 !} \ldots, \\
B_{2}=\frac{1}{6}, B_{4}=-\frac{1}{30}, B_{6}=\frac{1}{42}, B_{8}=-\frac{1}{30}, B_{10}=\frac{5}{66}, B_{12}=-\frac{691}{2730}, B_{14}=\frac{7}{6}, \ldots
\end{gathered}
$$

The most difficult part of Harer and Zagier's approach was probably their proof of the HarerZagier formula (1.7). In particular, they used integration over a Gaussian set of random matrices. Itzykson and Zuber [16] have simplified this with the help of harmonic oscillators and the Baker-Campbell-Hausdorff formula. A further simplification can be obtained by using matching polynomials (see [40], VI-34, remark 21, or [11], chapter 1) allowing to solve the integral (3.5) of [16] directly. Matrix integration is maybe the most popular method for this problem, see [30, $18,19,42,22,14,1]$, because those matrices can be diagonalized. As we have shown in [24], 
however, it is possible to replace the integrals by differential operators. Usually, differentiation is easier than integration, and in this particular case, it is even possible to diagonalize the differential operators in a combinatorial way using the so called BEST theorem (for counting directed Eulerian tours), as shown in [23], where we have given the first combinatorial proof of the Harer-Zagier formula (1.7). Goulden and Nica's proof [12] of this formula also uses implicitly the BEST theorem, but finishes by counting planar arborescences, not arborescences with marked vertices as [23].

Another technique useful for proving the Harer-Zagier formula (1.7) is representation theory, see $[17,31,16,41]$ and Zagier's appendix in [22]. This method, however, is based on the regular representation of the symmetric group $S_{n}$, in $n$ ! dimensions instead of $n$ dimensions. Therefore it is not necessarily the easiest approach.

Having calculated $\chi\left(\mathcal{M}_{g ; 1}\right)$, the orbifold Euler characteristic of the moduli space $\mathcal{M}_{g ; n}$ of genus $g$ smoth curves with $n$ marked points can be obtained using the recurrence relation

$$
\chi\left(\mathcal{M}_{g ; n+1}\right)=\chi\left(\mathcal{M}_{g ; n}\right)(2-2 g-n)
$$

for stable $(g, n)$. It follows from the facts that the forgetful mapping $\mathcal{M}_{g ; n+1} \rightarrow \mathcal{M}_{g ; n}$ is a fiber bundle whose fiber is a genus $g$ curve punctured at $n$ points, that the Euler characteristic of the fiber is $2-2 g-n$ and that the Euler characteristic of the total space (of an orbifold fiber bundle) is the product of the Euler characteristics of the base and of the fiber. This recurrence relation was exploited by Kontsevich [20] (see also [30,22]) in order to reduce the matrix integration to the one-dimensional case. Finally (1.8) follows from Stirling's formula for $\log \Gamma(t)$.

We want to give a short proof of (1.8) using only formal power series and classical combinatorics but no integration or representation theory. We hope that this will make combinatorial approaches even more popular than they are already $[4,5,6,7,8,9,12,13,21,26,27,28,29$, $32,33,37,38,39]$. We start in the same way as Harer and Zagier: We color the $v$ vertices of our embedded graph (obtained after gluing in pairs the oriented sides of our ( $2 m$ )-gon) in order to allow additional identifications among those vertices (vertices getting the same color). Harer and Zagier used $N$ colors and had to evaluate (1.7):

$$
\sum_{g=0}^{\lfloor m / 2\rfloor} \varepsilon_{g}(m) \cdot N^{m+1-2 g}=\sum_{g=0}^{\lfloor m / 2\rfloor} \varepsilon_{g}(m) \cdot N^{v} .
$$

Inspired by $[35,25]$, we use an infinite number of colors corresponding to an infinite number of variables $x_{1}, x_{2}, x_{3}, \ldots$, and each variable appears to the power of the degree of the vertex colored with it. In other words, if our $(2 m-1)$ !! gluings lead to $\varepsilon_{d_{1}, d_{2}, d_{3}, \ldots}(m)$ embedded graphs having $d_{1}$ vertices of degree $1, d_{2}$ vertices of degree $2, d_{3}$ vertices of degree 3 , etc., then we have to evaluate

$$
\sum_{d_{1}, d_{2}, d_{3}, \cdots \geq 0} \varepsilon_{d_{1}, d_{2}, d_{3}, \ldots}(m) \cdot p_{1}^{d_{1}} p_{2}^{d_{2}} p_{3}^{d_{3}} \ldots,
$$

where

$$
p_{1}=\sum_{i=1}^{\infty} x_{i}, \quad p_{2}=\sum_{i=1}^{\infty} x_{i}^{2}, \quad p_{3}=\sum_{i=1}^{\infty} x_{i}^{3}, \quad \ldots
$$

are the classical power symmetric functions. The elementary symmetric functions $e_{1}, e_{2}, e_{3}, \ldots$ can be defined by the identity

$$
1+\sum_{n=1}^{\infty} e_{n} t^{n}=\prod_{i=1}^{\infty}\left(1+x_{i} t\right)
$$


whereas the complete homogeneous symmetric functions $h_{1}, h_{2}, h_{3}, \ldots$ are given by

$$
1+\sum_{n=1}^{\infty} h_{n} t^{n}=\prod_{i=1}^{\infty}\left(1+x_{i} t+x_{i}^{2} t^{2}+x_{i}^{3} t^{3}+\ldots\right)=\prod_{i=1}^{\infty} \frac{1}{1-x_{i} t} .
$$

For every $m \geq 1, h_{m}$ admits a natural decomposition

$$
h_{m}=\sum_{n=1}^{m} h_{m, n}, \quad h_{m, 1}=p_{m}, \quad h_{m, m}=e_{m},
$$

into parts $h_{m, n}$ using exactly $n$ different variables (and thus interpolating between $p_{m}$ and $e_{m}$ ). Their generating function is

$$
\begin{aligned}
1+\sum_{1 \leq n \leq m} h_{m, n} u^{n} t^{m} & =\prod_{i=1}^{\infty}\left(1+x_{i} u t+x_{i}^{2} u t^{2}+x_{i}^{3} u t^{3}+\ldots\right)=\prod_{i=1}^{\infty} \frac{1-x_{i}(t-u t)}{1-x_{i} t} \\
& =\exp \left[\sum_{i=1}^{\infty} \log \left(1-x_{i}(t-u t)\right)-\log \left(1-x_{i} t\right)\right] \\
& =\exp \left[\sum_{i=1}^{\infty} \sum_{j=1}^{\infty} x_{i}^{j}\left(\frac{t^{j}}{j}-\frac{(t-u t)^{j}}{j}\right)\right] \\
& =\exp \left[\sum_{j=1}^{\infty} p_{j}\left(\frac{t^{j}}{j}-\frac{(t-u t)^{j}}{j}\right)\right]
\end{aligned}
$$

and allows to develop the symmetric functions $h_{m, n}$ in the basis of power symmetric functions $p_{j}$. We explain in the next section that our combinatorial proof [23] of the Harer-Zagier formula (1.7) also implies the following result.

Theorem 3. (Weighted Harer-Zagier formula) For fixed $m \in \mathbb{N}^{*}$ we have

$$
\frac{1}{2 m} \sum_{d_{1}, d_{2}, d_{3}, \cdots \geq 0} \varepsilon_{d_{1}, d_{2}, d_{3}, \ldots}(m) \cdot p_{1}^{d_{1}} p_{2}^{d_{2}} p_{3}^{d_{3}} \cdots=\sum_{n=1}^{m+1} h_{2 m, n} \cdot \frac{(2 m-n) !}{2^{m+1-n}(m+1-n) !} .
$$

Since 2001 we have presented this weighted version instead of the classical one in every seminar enamored of symmetric functions. Another proof for it can be found in [9]. In the last section we finally show how the weighted Harer-Zagier formula can be used to calculate the Euler characteristic of the moduli space of curves (1.8).

\section{Proof of the weighted Harer-Zagier formula}

Each coloration considered in the left hand side of (1.18) uses only a finite number $n$ of different colors, and without loss of generality, we can consider that the set of those colors really used is $V=\{1,2,3, \ldots, n\}$, the set of vertices of a multigraph (loops and multiple edges are allowed) with $m$ unoriented edges each of which was obtained by gluing two oppositely oriented arcs of our $2 m$-gon (as suggested by Berge [3], preface). This $2 m$-gon defines an oriented Eulerian circuit of the graph. The division of the left hand side by $2 m$ (the number of rotations of our 
$2 m$-gon) shows that we should consider that the arc of our $2 m$-gon labeled 1 starts at our vertex labeled 1 and uses a fixed outgoing arc from it. For every $v \in V \backslash\{1\}$ let us consider the unique last arc of our Eulerian circuit (the arc with the heighest label) leaving it. Those $b=n-1$ arcs define an (oriented) arborescence

$$
a: V \backslash\{1\} \rightarrow V
$$

with root 1 ( $a$ has no cycles and iterating $a$ leads to 1 , see [10], page 61). Moreover, our oriented Eulerian circuit defines for every $v \in V$ an order among the other arcs (not belonging to the arborescence $a$ ) leaving $v$ : the order in which it uses those arcs. On the other hand, given the arborescence $a$ and, for every $v \in V$, the order of other departs, we uniquely reconstruct our Eulerian circuit (this is the content of the theorem called BEST, see [34], theorem 5.6.2 or [3], chapter 11.3, theorem 8).

Let us associate to each $v \in V$ the variable $x_{v}$ (used in our symmetric function) and the additional variable $y_{v}$ (used for counting purposes only). It is classical to associate to the arborescence $a: V \backslash\{1\} \rightarrow V$ the monomial

$$
a(\mathbf{x}, \mathbf{y})=\prod_{v \in V \backslash\{1\}} x_{a(v)} y_{a(v)} .
$$

According to Prüfer and Foata, it is natural to code the function $a: V \backslash\{1\} \rightarrow V$ by the sequence of its $b=n-1$ values $a\left(v_{1}\right), a\left(v_{2}\right), \ldots, a\left(v_{b}\right)$. For $v_{1}$ we can take $v_{1}=\min [V \backslash a(V \backslash\{1\})]$. Since $a^{\prime}=\left.a\right|_{V \backslash v_{1}}$ is still an arborescence, we can apply the same procedure to $a^{\prime}$ obtaining finally a bijection between arborescences $a: V \backslash\{1\} \rightarrow V$ and number sequences $a\left(v_{1}\right) \in V, a\left(v_{2}\right) \in V$, $\ldots, a\left(v_{b-1}\right) \in V, a\left(v_{b}\right)=1$ such that $a(\mathbf{x}, \mathbf{y})=x_{a\left(v_{1}\right)} y_{a\left(v_{1}\right)} \ldots x_{a\left(v_{b}\right)} y_{a\left(v_{b}\right)}$. This proves Cayley's theorem on the weighted counting of arborescences (see [34], chapter 5.3):

$$
\sum_{a: V \backslash\{1\} \rightarrow V} a(\mathbf{x}, \mathbf{y})=\left(x_{1} y_{1}+\cdots+x_{n} y_{n}\right)^{b-1} \cdot x_{1} y_{1} .
$$

Among our $m$ unoriented edges, the arborescence $a$ uses (and distinguishes) $b=n-1$. If they create at the vertex $v \in V$ the degree $d_{B}(v)$, then

$$
\frac{a(\mathbf{x}, \mathbf{y})}{x_{1} y_{1}}=\prod_{v \in V}\left(x_{v} y_{v}\right)^{d_{B}(v)-1}
$$

Let us code the $s=m-b$ supplementary undirected edges by a multiset $S: V \cup\left(\begin{array}{c}V \\ 2\end{array}\right) \rightarrow \mathbb{N}$, where $S(v)$ denotes the number of loops at $v \in V$ and $S(\{u, v\})$ denotes the number of edges between the two distinct vertices $u, v \in V$. Of course,

$$
|S|=\sum_{v \in V} S(v)+\sum_{\{u, v\} \in\left(\begin{array}{c}
V \\
2
\end{array}\right)} S(\{u, v\})=s=m-b=m+1-n,
$$

and the supplementary degrees for each $v \in V$ are

$$
d_{S}(v)=2 \cdot S(v)+\sum_{u \in V \backslash\{v\}} S(\{u, v\}) .
$$

The contribution for our symmetric function (1.18) is now

$$
\prod_{v \in V} x_{v}^{d_{B}(v)+d_{S}(v)},
$$


but, since an Eulerian circuit is coded by its arborescence and, for every $v \in V$, the order of other departs, we have to multiply by

$$
\prod_{v \in V}\left(d_{B}(v)-1+d_{S}(v)\right) !=\sum_{\substack{i_{1}+\cdots+i_{n}=b-1+2 s \\ i_{1}, \ldots, i_{n} \geq 0}} \partial_{y_{1}}^{i_{1}} \ldots \partial_{y_{n}}^{i_{n}} \prod_{v \in V} y_{v}^{d_{B}(v)-1+d_{S}(v)} .
$$

Indeed, at every vertex different from 1 there is one edge of the arborescence which must not be taken into account in the order of other departs, and at the vertex 1 , there is the edge 1 which must not be taken into account. Finally, the contribution of $V=\{1, \ldots, n\}$ to (1.18) is

$$
\prod_{v \in V} x_{v} \sum_{\substack{i_{1}+\cdots+i_{n}=b-1+2 s \\
i_{1}, \ldots, i_{n} \geq 0}} \partial_{y_{1}}^{i_{1}} \ldots \partial_{y_{n}}^{i_{n}} \frac{a(\mathbf{x}, \mathbf{y})}{x_{1} y_{1}} \prod_{v \in V} \frac{\left[\frac{\left(x_{v} y_{v}\right)^{2}}{2}\right]^{S(v)}}{S(v) !} \prod_{\{u, v\} \in\left(\begin{array}{c}
V \\
2
\end{array}\right)} \frac{\left[\left(x_{u} y_{u}\right)\left(x_{v} y_{v}\right)\right]^{S(\{u, v\})}}{S(\{u, v\}) !}
$$

summed over all arborescences $a: V \backslash\{1\} \rightarrow V$ and over all multisets $S: V \cup\left(\begin{array}{l}V \\ 2\end{array}\right) \rightarrow \mathbb{N}$ with $|S|=s$ (the divisions by $S(\{u, v\})$ !, by $S(v)$ ! and by 2 reflect the facts that we must not distinguish Eulerian circuits obtained from each other by permutations of supplementary multiple edges, supplementary multiple loops or arcs within a loop). Using (2.3) and

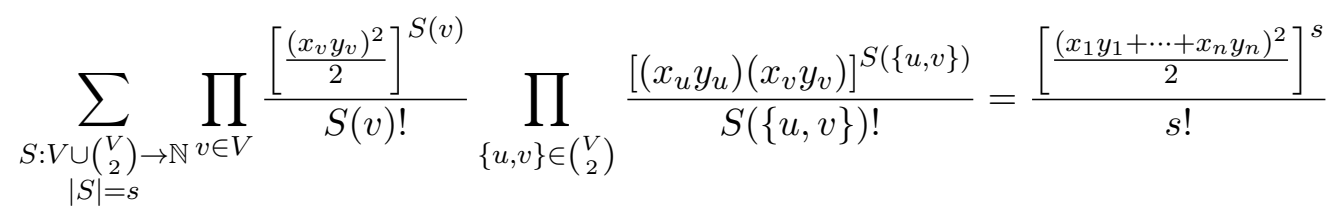

the contribution of $V=\{1, \ldots, n\}$ to (1.18) becomes

$$
\begin{aligned}
& \prod_{v \in V} x_{v} \sum_{\substack{i_{1}+\ldots+i_{n}=b-1+2 s \\
i_{1}, \ldots, i_{n} \geq 0}} \partial_{y_{1}}^{i_{1}} \ldots \partial_{y_{n}}^{i_{n}} \frac{\left(x_{1} y_{1}+\cdots+x_{n} y_{n}\right)^{b-1+2 s}}{2^{s} s !} \\
= & \frac{(b-1+2 s) !}{2^{s} s !} \sum_{\substack{i_{1}+\cdots+i_{n}=b-1+2 s \\
i_{1}, \ldots, i_{n} \geq 0}} x_{1}^{i_{1}+1} \ldots x_{n}^{i_{n}+1} .
\end{aligned}
$$

This finishes the proof of (1.18) because $\left(i_{1}+1\right)+\cdots+\left(i_{n}+1\right)=n+b-1+2 s=2(b+s)=2 m$, $b-1+2 s=2 m-n$ and $s=m-b=m+1-n$.

\section{The Euler characteristic of the moduli space of curves}

In order to establish (1.8) we have to prove that

$$
\begin{aligned}
\chi(z) & =\sum_{g=1}^{\infty} \chi\left(\mathcal{M}_{g ; 1}\right) \frac{z^{2 g-1}}{(2 g-1) !}=\sum_{g=1}^{\infty}-\frac{B_{2 g}}{2 g} \frac{z^{2 g-1}}{(2 g-1) !} \\
& =-\frac{1}{z} \sum_{k=2}^{\infty} B_{k} \frac{z^{k}}{k !}=-\frac{1}{z}\left[\frac{z}{e^{z}-1}+\frac{1}{2} z-1\right] \\
& =-\frac{1}{2}+\frac{1}{z}+\frac{1}{1-e^{z}} .
\end{aligned}
$$


Using (1.5) and (1.18) we know that $\chi(z)$ can be obtained from (1.17) if we replace $p_{1}$ and $p_{2}$ by 0 (in order to forbid vertices of degre 0 or 1 ) and $p_{j}$ by $x$ for every $j \geq 3$. This gives the expression

$$
\exp \left[x\left(-\log (1-t)+\log (1-t+u t)-u t-u t^{2}+\frac{u^{2} t^{2}}{2}\right)\right],
$$

where we have to replace each coefficient of $x^{v} u^{n} t^{2 m}$ by

$$
(-1)^{m-1} \frac{(2 m-n) !}{2^{m+1-n}(m+1-n) !} \cdot \frac{z^{m-v}}{(m-v) !},
$$

because $m-v=2 g-1 \geq 1$. Therefore we have

$$
\begin{aligned}
& \chi(z)= \sum_{m=1}^{\infty} \sum_{n=0}^{m+1} \sum_{v=0}^{m}(-1)^{m-1} \frac{(2 m-n) !}{2^{m+1-n}(m+1-n) !} \cdot \frac{z^{m-v}}{(m-v) !}\left\langle x^{v} u^{n} t^{2 m}\right\rangle \\
& \exp \left[x\left(-\log (1-t)+\log (1-t+u t)-u t-u t^{2}+\frac{u^{2} t^{2}}{2}\right)\right] \\
&= \sum_{m=1}^{\infty} \sum_{n=0}^{m+1}(-1)^{m-1} \frac{(2 m-n) !}{2^{m+1-n}(m+1-n) !}\left\langle x^{m} u^{n} t^{2 m}\right\rangle \\
& \exp [x z] \cdot \exp \left[x\left(-\log (1-t)+\log (1-t+u t)-u t-u t^{2}+\frac{u^{2} t^{2}}{2}\right)\right] \\
&=-\frac{1}{2} \sum_{m=1}^{\infty} \sum_{n=0}^{m+1}\left(\begin{array}{c}
2 m-n \\
m-1
\end{array}\right) \frac{1}{m}\left\langle u^{n} t^{2 m}\right\rangle \\
& {\left[-\frac{z}{2}+\frac{1}{2} \log (1-t)-\frac{1}{2} \log (1-t+2 u t)+u t+u t^{2}-u^{2} t^{2}\right]^{m} } \\
&=-\frac{1}{2} \sum_{m=1}^{\infty} \sum_{n=0}^{m+1}\left(\begin{array}{c}
2 m-n \\
m-1
\end{array}\right) \frac{1}{m}\left\langle u^{2 m-n} t^{2 m}\right\rangle \\
& {\left[-\frac{z}{2}+\frac{1}{2} \log (1-u t)-\frac{1}{2} \log (1-u t+2 t)+t+u t^{2}-t^{2}\right]^{m} } \\
&=-\frac{1}{2} \sum_{m=1}^{\infty} \frac{1}{m}\left\langle u^{m-1} t^{2 m}\right\rangle \\
& {\left[-\frac{z}{2}+\frac{1}{2} \log (1-(u+1) t)-\frac{1}{2} \log (1-(u+1) t+2 t)+t+(u+1) t^{2}-t^{2}\right]^{m} } \\
&=-\frac{1}{2} \sum_{m=1}^{\infty} \frac{1}{m}\left\langle u^{m-1} t^{m+1}\right\rangle\left[-\frac{z}{2}+\frac{1}{2} \log (1-u-t)-\frac{1}{2} \log (1-u+t)+t+u t\right]^{m} \\
&=-\frac{1}{2}\langle t\rangle \sum_{m=1}^{\infty} \frac{1}{m}\left\langle u^{-1}\right\rangle\left[\frac{z}{-\frac{z}{2}+\frac{1}{2} \log (1-u-t)-\frac{1}{2} \log (1-u+t)+t+u t}\right]^{-m} .(3.4 \\
&
\end{aligned}
$$

The formal power series in the variable $u$

$$
f_{z, t}(u)=\frac{u t}{-\frac{z}{2}+\frac{1}{2} \log (1-u-t)-\frac{1}{2} \log (1-u+t)+t+u t}
$$


whose coefficients are formal power series in $z$ (with coefficients in the field of formal Laurent series in $t$ ) has valuation 1, i.e. $\left\langle u^{0}\right\rangle f_{z, t}(u)=0$ and $\left\langle u^{1}\right\rangle f_{z, t}(u)$ is invertible. Therefore it admits a unique inverse $g_{z, t}(u)$ with respect to composition. Indeed, every formal power series $f(u)$ with $\left\langle u^{0}\right\rangle f(u)=0$ and $\left\langle u^{1}\right\rangle f(u)$ invertible has a unique left inverse and a unique right inverse with respect to composition as can be seen by comparing coefficients or by using [2]. By associativity of composition, these inverses are identical, i.e. we have a unique $g(u)$ such that $f(g(u))=g(f(u))=u$. Moreover, it is classical from residue calculus and easy to see directly that for any formal Laurent series $l(u)$ and any formal power series $p(u)$ of valuation $d>0$ (such that $\left\langle u^{d}\right\rangle p(u)$ is invertible) we have

$$
d \cdot\left\langle u^{-1}\right\rangle l(u)=\left\langle u^{-1}\right\rangle\left[l(p(u)) \cdot p^{\prime}(u)\right] .
$$

Using these results we can continue our calculation:

$$
\chi(z)=-\frac{1}{2}\langle t\rangle \sum_{m=1}^{\infty} \frac{1}{m}\left\langle u^{-1}\right\rangle u^{-m} \cdot \frac{\partial}{\partial u} g_{z, t}(u)=-\frac{1}{2}\langle t\rangle \sum_{m=1}^{\infty}\left\langle u^{m}\right\rangle g_{z, t}(u)=-\frac{1}{2}\langle t\rangle g_{z, t}(1) .
$$

But we know

$$
\begin{aligned}
f_{z, t}\left(g_{z, t}(1)\right) & =1 \Leftrightarrow \\
t g_{z, t}(1) & =-\frac{z}{2}+\frac{1}{2} \log \left(1-g_{z, t}(1)-t\right)-\frac{1}{2} \log \left(1-g_{z, t}(1)+t\right)+t+t g_{z, t}(1) \quad \Leftrightarrow \\
e^{z-2 t} & =\frac{1-g_{z, t}(1)-t}{1-g_{z, t}(1)+t} \Leftrightarrow 1-e^{z-2 t}=\frac{2 t}{1-g_{z, t}(1)+t} \Leftrightarrow \\
g_{z, t}(1) & =1+t-\frac{2 t}{1-e^{z-2 t}} .
\end{aligned}
$$

Therefore

$$
\begin{aligned}
\chi(z) & =-\frac{1}{2}+\left\langle t^{0}\right\rangle \frac{1}{1-e^{z-2 t}}=-\frac{1}{2}+\left\langle t^{0}\right\rangle\left[\frac{1}{z-2 t}+\frac{1}{1-e^{z-2 t}}\right] \\
& =-\frac{1}{2}+\frac{1}{z}+\frac{1}{1-e^{z}},
\end{aligned}
$$

because

$$
\frac{1}{z-2 t}=-\frac{1}{2 t} \cdot \frac{1}{1-\frac{z}{2 t}}=-\frac{1}{2 t}-\frac{z}{4 t^{2}}-\frac{z^{2}}{8 t^{3}}-\ldots
$$

as a formal power series in $z$ has no constant term in $t$, but

$$
\frac{1}{z-2 t}+\frac{1}{1-e^{z-2 t}}
$$

is a formal power series in both $z$ and $t$, so its constant term in $t$ is obtained by putting $t=0$. This concludes our proof.

Acknowledgements: We would like to thank Gerd Mersmann for an inspiring introduction to formal power series. This work was supported by the LABEX MILYON (ANR-10-LABX-0070) of Université de Lyon, within the program "Investissements d'Avenir" (ANR-11-IDEX-0007) operated by the French National Research Agency (ANR). 


\section{References}

[1] A. Aggarwal, An arithmetic property of moments of the $\beta$-Hermite ensemble and certain map enumerators, Electron. J. Combin. 25 no. 1 (2018), Paper 1.29, 33 pp.

[2] R. Bacher and B. Lass, Développements limités et réversion des séries [Taylor expansions and power series reversion], Enseign. Math. (2) 52 no. 3-4 (2006), 267-293.

[3] C. Berge, Graphes [Graphs], Dunod, Paris, 1983.

[4] O. Bernardi, An analogue of the Harer-Zagier formula for unicellular maps on general surfaces, Adv. in Appl. Math. 48 no. 1 (2012), 164-180.

[5] O. Bernardi and G. Chapuy, Counting unicellular maps on non-orientable surfaces, Adv. in Appl. Math. 47 no. 2 (2011), 259-275.

[6] O. Bernardi and G. Chapuy, A bijection for covered maps, or a shortcut between HarerZagier's and Jackson's formulas. J. Combin. Theory Ser. A 118 no. 6 (2011), 1718-1748.

[7] O. Bernardi and A. H. Morales, Bijections and symmetries for the factorizations of the long cycle, Adv. in Appl. Math. 50 no. 5 (2013), 702-722.

[8] G. Chapuy, A new combinatorial identity for unicellular maps, via a direct bijective approach, Adv. in Appl. Math. 47 no. 4 (2011), 874-893.

[9] G. Chapuy, V. Féray, and E. Fusy, A simple model of trees for unicellular maps, J. Combin. Theory Ser. A 120 no. 8 (2013), 2064-2092.

[10] D. Foata and M.-P. Schützenberger, Théorie géométrique des polynômes eulériens, Lecture Notes in Mathematics, Vol. 138, Springer-Verlag, Berlin-New York, 1970.

[11] C. D. Godsil, Algebraic combinatorics, Chapman and Hall Mathematics Series, Chapman \& Hall, New York, 1993.

[12] I. P. Goulden and A. Nica, A direct bijection for the Harer-Zagier formula, J. Combin. Theory Ser. A 111 no. 2 (2005), 224-238.

[13] I. P. Goulden and W. Slofstra, Annular embeddings of permutations for arbitrary genus, J. Combin. Theory Ser. A 117 no. 3 (2010), 272-288.

[14] U. Haagerup and S. Thorbjørnsen, Random matrices with complex Gaussian entries, Expo. Math. 21 no. 4 (2003), 293-337.

[15] J. Harer and D. Zagier, The Euler characteristic of the moduli space of curves, Invent. Math. 85 no. 3 (1986), 457-485.

[16] C. Itzykson and J. B. Zuber, Matrix integration and combinatorics of modular groups, Comm. Math. Phys. 134 no. 1 (1990), 197-207.

[17] D. M. Jackson, Counting cycles in permutations by group characters, with an application to a topological problem, Trans. Amer. Math. Soc. 299 no. 2 (1987), 785-801. 
[18] D. M. Jackson, On an integral representation for the genus series for 2-cell embeddings, Trans. Amer. Math. Soc. 344 no. 2 (1994), 755-772.

[19] S. V. Kerov, Rook placements on Ferrers boards, and matrix integrals, Zap. Nauchn. Sem. S.-Peterburg. Otdel. Mat. Inst. Steklov. (POMI) 240 (1997), Teor. Predst. Din. Sist. Komb. i Algoritm. Metody. 2, 136-146, 293, translation in J. Math. Sci. (New York) 96 no. 5 (1999), 3531-3536.

[20] M. Kontsevich, Intersection theory on the moduli space of curves and the matrix Airy function, Comm. Math. Phys. 147 no. 1 (1992), 1-23.

[21] S. K. Lando, Lekcii o proizvodyashchih funkciyah, MCNMO, Moskva, 2007.

[22] S. K. Lando and A. K. Zvonkin, Graphs on surfaces and their applications, with an appendix by Don B. Zagier, Encyclopaedia of Mathematical Sciences, 141, Low-Dimensional Topology, II, Springer-Verlag, Berlin, 2004.

[23] B. Lass, Démonstration combinatoire de la formule de Harer-Zagier [A combinatorial proof of the Harer-Zagier formula], C. R. Acad. Sci. Paris Sér. I Math. 333 no. 3 (2001), 155-160.

[24] B. Lass, The $N$-dimensional matching polynomial, Geom. Funct. Anal. 15 no. 2 (2005), 453-475.

[25] B. Lass, Orientations acycliques et le polynôme chromatique [Acyclic orientations and the chromatic polynomial], European J. Combin. 22 no. 8 (2001), 1101-1123.

[26] M. A. Ledoux, A recursion formula for the moments of the Gaussian orthogonal ensemble, Ann. Inst. Henri Poincaré Probab. Stat. 45 no. 3 (2009), 754-769.

[27] V. Liskovets, A note on the total number of double Eulerian circuits in multigraphs, J. Integer Seq. 5 no. 2 (2002), Article 02.2.5, 3 pp.

[28] A. H. Morales and E. A. Vassilieva, Direct bijective computation of the generating series for 2 and 3-connection coefficients of the symmetric group, Electron. J. Combin. 20 no.2 (2013), Paper 6, 27 pp.

[29] A. Morozov and Sh. Shakirov, Exact 2-point function in Hermitian matrix model, J. High Energy Phys. no. 12 (2009), 003, 33 pp.

[30] R. C. Penner, Perturbative series and the moduli space of Riemann surfaces, J. Differential Geom. 27 no. 1 (1988), 35-53.

[31] B. Pittel, Another proof of the Harer-Zagier formula, Electron. J. Combin. 23 no. 1 (2016), Paper 1.21, $11 \mathrm{pp}$.

[32] G. Schaeffer and E. A. Vassilieva, A bijective proof of Jackson's formula for the number of factorizations of a cycle, J. Combin. Theory Ser. A 115 no. 6 (2008), 903-924.

[33] R. Speicher, Zufallsmatrizen, das Zählen von Flächen und freie Wahrscheinlichkeitstheorie. [Random matrices, the counting of surfaces and free probability theory], Mitt. Dtsch. Math.Ver. 23 no. 1 (2015), 36-42. 
[34] R. P. Stanley, Enumerative combinatorics, Vol. 2, with a foreword by Gian-Carlo Rota and appendix 1 by Sergey Fomin, Cambridge Studies in Advanced Mathematics, 62, Cambridge University Press, Cambridge, 1999.

[35] R. P. Stanley, A symmetric function generalization of the chromatic polynomial of a graph, Adv. Math. 111 no. 1 (1995), 166-194.

[36] K. Strebel, Quadratic differentials, Springer-Verlag, Berlin, 1984.

[37] E. A. Vassilieva, Moments of normally distributed random matrices given by generating series for connection coefficients - explicit bijective computation, Ann. Comb. 21 no. 3 (2017), 445-477.

[38] E. A. Vassilieva, Bijective enumeration of 3-factorizations of an N-cycle, Ann. Comb. 16 no. 2 (2012), 367-387.

[39] E. A. Vassilieva and G. Schaeffer, A combinatorial way of counting unicellular maps and constellations (Russian), Fundam. Prikl. Mat. 17 no. 4 (2011/12), 25-52; translation in J. Math. Sci. (N.Y.) 191 no. 5 (2013), 613-632.

[40] G. Viennot, Une théorie combinatoire des polynômes orthogonaux généraux, Notes de conférences données à l'Université du Québec à Montréal, 1983.

[41] D. Zagier, On the distribution of the number of cycles of elements in symmetric groups, Nieuw Arch. Wisk. (4) 13 no. 3 (1995), 489-495.

[42] A. Zvonkin, Matrix integrals and map enumeration: an accessible introduction, Combinatorics and physics (Marseille, 1995), Math. Comput. Modelling 26 no. 8-10 (1997), 281-304. 